\title{
Article
}

\section{Strength training for people with multiple sclerosis and the current recommendations}

Gooch, Helen, Hill, James Edward and Clegg, Andrew

Available at http://clok.uclan.ac.uk/39008/

Gooch, Helen, Hill, James Edward ORCID: 0000-0003-1430-6927 and Clegg, Andrew ORCID: 0000-0001-8938-7819 (2021) Strength training for people with multiple sclerosis and the current recommendations. British Journal of Neuroscience Nursing, 17 (S3). S32-S41. ISSN 1747-0307

It is advisable to refer to the publisher's version if you intend to cite from the work. 10.12968/bjnn.2021.17.sup3.s32

For more information about UCLan's research in this area go to http://www.uclan.ac.uk/researchgroups/ and search for <name of research Group>.

For information about Research generally at UCLan please go to http://www.uclan.ac.uk/research/

All outputs in CLoK are protected by Intellectual Property Rights law, including Copyright law. Copyright, IPR and Moral Rights for the works on this site are retained by the individual authors and/or other copyright owners. Terms and conditions for use of this material are defined in the policies page.

\section{CLoK}

Central Lancashire online Knowledge www.clok.uclan.ac.uk

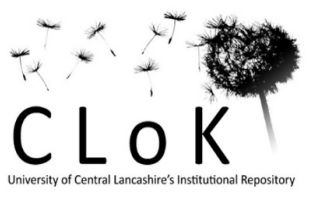


Commentary on: Strength training for people with multiple sclerosis and the current recommendations.

\section{ABSTRACT}

Recent guidelines recommend strength and conditioning training for patients with multiple sclerosis (MS). This article evaluates and summarises four systematic reviews examining strength training for people with MS and examines if these reviews substantiate these current guidelines.

\section{Papers being reviewed}

- Cruickshank TM, Reyes AR, Ziman MR. A systematic review and meta-analysis of strength training in individuals with multiple sclerosis or Parkinson disease. Medicine. 2015;94(4):1-15. https://doi.org/10.1097/MD.0000000000000411

- Jørgensen M, Dalgas U, Wens I, Hvid LG. Muscle strength and power in persons with multiple sclerosis - a systematic review and meta-analysis. J Neurol Sci. 2017;376:225-241. https://doi.org/10.1016/j.jns.2017.03.022

- Mañago MM, Glick S, Hebert JR, Coote S, Schenkman M. (2019). Strength Training to Improve Gait in People with Multiple Sclerosis: A Critical Review of Exercise Parameters and Intervention Approaches. Int J MS Care. 2019;21(2):47-56. https://doi.org/10.7224/1537-2073.2017-079

- Manca A, Dvir Z, Deriu F. Meta-analytic and Scoping Study on Strength Training in People With Multiple Sclerosis. J Strength Cond Res. 2019;33(3):874-889. https://doi.org/10.1519/JSC.0000000000002381

\section{Background to the reviews}

Multiple sclerosis (MS) is estimated to affect 2.8 million individuals worldwide (Walton et al, 2020), with 105780 individuals living with the condition in England (Public Health England (PHE), 2020). People with MS (PwMS) can experience a variety of symptoms, including physical impairments, fatigue, pain and cognitive deficits, which often result in a progressive limitation of function (Amatya et al, 2019) and have a negative impact on quality of life (Jones et al, 2008). MS also has a substantial economic impact on health care services (Kobelt et al,, 2017), especially as diagnosis is frequently in early-to-middle adulthood (Walton et al, 2020) and life expectancy is only reduced by around 7 years (Lunde et al, 2017).

Exercise and physical activity are reported as a key component of the management strategies of PwMS and has been shown to improve functional outcomes, improve quality of life and reduce fatigue (Amatya et al, 2019). However, PwMS participate in significantly lower levels of exercise and activity compared to those without a long-term condition or disability (Kinnett-Hopkins et al, 2017). The updated UK physical activity guidelines (PHE, 2019) have, for the first time, included guidelines for disabled adults, supported by a rapid evidence review (PHE, 2018), evaluating physical activity for a broad range of impairments. 
However, it is important that clinicians use these guidelines in the context of condition specific recommendations (Latimer-Cheung et al, 2013).

The updated UK physical activity guidelines also place an increased focus on the benefits of strength training (PHE, 2019). Strength training has been shown to improve muscle strength in PwMS, although its impact on balance, functional outcomes and quality of life is less clear (Kjølhede et al, 2012). Over the last decade, two guidelines have been developed that facilitate the implementation of strength training specifically for PwMS (Latimer-Cheung et al, 2013a; Kim et al, 2019). The guideline produced by Latimer-Cheung et al, (2013a) was informed by a systematic review and descriptive data analysis (Latimer-Cheung et al, 2013b), alongside an expert and stakeholder review. It identified that there was adequate evidence to formulate a guideline to achieve fitness benefits; however, the evidence was insufficient to formulate guidelines to inform improvements in mobility, fatigue or quality of life. Kim et al, (2019) developed a guideline through a synthesis of nine previous guidelines, dated between 1999 and 2017, including the guideline by Latimer-Cheung et al, (2013b). Many of these synthesised guidelines are based on review articles and lack evidence of a systematic approach to the guideline development. The two sets of exercise guidelines focus on adults living with mild to moderate MS and have many commonalities, providing a starting point for clinicians and PwMS to explore strength training. However, four systematic reviews (Cruickshank et al, 2015; Jørgensen et al, 2017; Mañago et al, 2019; Manca et al, 2019) relating to strength training for PwMS have been published after the Latimer-Cheung et al (2013b) guidelines and are not referenced within the guidelines by Kim et al (2019).

The aim of this commentary is to:

1. Critically appraise the methods used within these four systematic reviews and report the key findings on strength training for PwMS

2. Cross-reference the guideline recommendations against relevant studies (studies that have used similar methods as recommended in the guidelines) within the four systematic reviews in order to verify, challenge or develop the key points of the guidelines for strength training for PwMS. 


\section{Methods and quality of the reviews}

The core inclusion criteria for all four reviews were studies of PwMS who had undergone strength training and were assessed with a muscle strength outcome. All four reviews included randomised controlled trials (RCTs), with two reviews also including nonrandomised trials (Cruickshank et al, 2015; Jørgensen et al, 2017). Two reviews (Jørgensen et al, 2017; Manca et al, 2019) only included studies with a no exercise training control. See Table 1 for the full list of Population, Intervention, Control, Outcomes (PICO) variables.

Table 2. Population, Intervention, Control, Outcomes (PICO) characteristics

\begin{tabular}{|c|c|c|c|c|}
\hline PICO & $\begin{array}{c}\text { Cruickshank et al } \\
\text { (2015) }\end{array}$ & $\begin{array}{l}\text { Jørgensen et al } \\
\text { (2017) }\end{array}$ & Manca et al (2019) & Mañago et al (2019) \\
\hline Population & Diagnosis of MS & Diagnosis of MS & Diagnosis of MS & Diagnosis of MS \\
\hline Intervention & Strength training & Strength training & Strength training & $\begin{array}{l}\text { Strength training for } \\
\text { lower extremities } \\
\text { and /or trunk } \\
\text { included in study } \\
\text { but not necessarily } \\
\text { primary } \\
\text { intervention. }\end{array}$ \\
\hline Control & Not specified & $\begin{array}{l}\text { No training or usual } \\
\text { care }\end{array}$ & $\begin{array}{l}\text { No intervention or } \\
\text { assigned to a } \\
\text { waiting list }\end{array}$ & $\begin{array}{l}\text { Inactive or other } \\
\text { active intervention }\end{array}$ \\
\hline Outcomes & $\begin{array}{l}\text { Inclusion } \\
\text { outcomes: } \\
\text { Muscle strength } \\
\text { (1RM, } \\
\text { maximum } \\
\text { voluntary } \\
\text { isometric } \\
\text { contraction, } \\
\text { maximum } \\
\text { voluntary dynamic } \\
\text { contraction, } \\
\text { power) } \\
\text { Other outcomes: } \\
\text { Functional } \\
\text { Mobility, Balance, } \\
\text { Functional } \\
\text { Capacity, Quality } \\
\text { of Life, Fatigue, } \\
\text { Mood, Muscle } \\
\text { endurance }\end{array}$ & $\begin{array}{l}\text { Inclusion outcomes: } \\
\text { Muscle strength } \\
\text { (1RM, } \\
\text { maximum voluntary } \\
\text { isometric } \\
\text { contraction, } \\
\text { maximum voluntary } \\
\text { dynamic } \\
\text { contraction, power) } \\
\end{array}$ & $\begin{array}{l}\text { Inclusion outcomes: } \\
\text { Muscle strength } \\
\text { (1 repetition } \\
\text { maximum [1RM], } \\
\text { maximal voluntary } \\
\text { isometric } \\
\text { contraction) } \\
\text { Other outcomes: } \\
\text { Gait performance } \\
\text { Balance } \\
\text { Fatigue, Function } \\
\text { Quality of Life }\end{array}$ & $\begin{array}{l}\text { Inclusion outcomes: } \\
\text { Muscle strength } \\
\text { (1RM, maximal } \\
\text { force, endurance) } \\
\text { Gait performance }\end{array}$ \\
\hline
\end{tabular}

Table note: $\mathrm{MS}=$ multiple sclerosis 
All four studies were judged to be methodologically robust, using the Joanna Briggs Institute (JBI) critical appraisal checklist for systematic reviews and research syntheses (Aromataris et al, 2015). The only areas of concern were the methods of synthesis and assessment of publication bias for Mañago et al (2019) and the data extraction and search strategy for Jørgensen et al (2017). Table 2 features quality assessment and corresponding methods for all of the systematic reviews.

Table 2. Quality assessment (JBI) and methods

\begin{tabular}{|c|c|c|c|c|}
\hline Criteria & $\begin{array}{c}\text { Cruickshank et al } \\
\text { (2015) }\end{array}$ & $\begin{array}{l}\text { Jørgensen et al } \\
\text { (2017) }\end{array}$ & $\begin{array}{l}\text { Manca et al } \\
\text { (2019) }\end{array}$ & $\begin{array}{l}\text { Mañago et al } \\
\text { (2019) }\end{array}$ \\
\hline $\begin{array}{l}\text { 1. Is the review } \\
\text { question clearly } \\
\text { and explicitly } \\
\text { stated? }\end{array}$ & $\begin{array}{c}\text { Yes: } \\
\text { To examine the } \\
\text { effects and } \\
\text { response } \\
\text { differences to } \\
\text { strength training } \\
\text { between people } \\
\text { with Parkinson's } \\
\text { disease or PwMS }\end{array}$ & $\begin{array}{c}\text { Yes: } \\
\text { To examine } \\
\text { effects of } \\
\text { progressive } \\
\text { resistance } \\
\text { training on } \\
\text { muscle function } \\
\text { for PwMS }\end{array}$ & $\begin{array}{c}\text { Yes: } \\
\text { To identify } \\
\text { changes to } \\
\text { muscle strength } \\
\text { and functional } \\
\text { outcomes after } \\
\text { strength training } \\
\text { in PwMS }\end{array}$ & $\begin{array}{c}\text { Yes: } \\
\text { To appraise the } \\
\text { exercise } \\
\text { parameters and } \\
\text { intervention of } \\
\text { strength training } \\
\text { as a means to } \\
\text { improve walking } \\
\text { in PwMS }\end{array}$ \\
\hline $\begin{array}{l}\text { 2. Were the } \\
\text { inclusion criteria } \\
\text { appropriate for } \\
\text { the review } \\
\text { question? }\end{array}$ & $\begin{array}{c}\text { Yes: } \\
\text { Included key } \\
\text { aspects of PICO } \\
\text { criteria }\end{array}$ & $\begin{array}{c}\text { Yes: } \\
\text { Included key } \\
\text { aspects of PICO } \\
\text { criteria }\end{array}$ & $\begin{array}{c}\text { Yes: } \\
\text { Included key } \\
\text { aspects of PICO } \\
\text { criteria. } \\
\text { Only RCTs } \\
\text { included }\end{array}$ & $\begin{array}{c}\text { Yes: } \\
\text { Included key } \\
\text { aspects of PICO } \\
\text { criteria. } \\
\text { Only RCTs } \\
\text { included }\end{array}$ \\
\hline $\begin{array}{l}\text { 3. Was the search } \\
\text { strategy } \\
\text { appropriate? }\end{array}$ & \begin{tabular}{|c|} 
Yes: \\
Full description of \\
the search \\
strategy. \\
Relevant key \\
terms used. \\
No additional \\
filters were \\
applied \\
\end{tabular} & $\begin{array}{l}\text { No: } \\
\text { No description } \\
\text { given of the } \\
\text { search strategy }\end{array}$ & $\begin{array}{c}\text { Yes: } \\
\text { Full description of } \\
\text { the search } \\
\text { strategy. Relevant } \\
\text { key terms used. } \\
\text { Limited to English } \\
\text { language only }\end{array}$ & $\begin{array}{l}\text { Yes: } \\
\text { Full description of } \\
\text { the search } \\
\text { strategy. } \\
\text { Relevant key } \\
\text { terms used. } \\
\text { Limited to English } \\
\text { language only }\end{array}$ \\
\hline $\begin{array}{l}\text { 4. Were the } \\
\text { sources and } \\
\text { resources used to } \\
\text { search for studies } \\
\text { adequate? }\end{array}$ & \begin{tabular}{|c|} 
Yes: \\
Multi-database \\
search (from date \\
of inception to \\
July 2014). \\
No methods of \\
identification of \\
additional studies \\
were used. \\
No description of \\
screening process
\end{tabular} & \begin{tabular}{|c|} 
Yes: \\
Multi-database \\
search (date of \\
inception to \\
March 2016). \\
Reference lists of \\
included studies \\
were checked. \\
Screening was \\
conducted by one \\
author after \\
completing an
\end{tabular} & $\begin{array}{c}\text { Yes: } \\
\text { Multi-database } \\
\text { search (date of } \\
\text { inception to May } \\
\text { 2017). } \\
\text { Scrutiny of clinical } \\
\text { trials registers. } \\
\text { Reference lists of } \\
\text { included studies } \\
\text { were checked. } \\
\text { Screening was } \\
\text { conducted by two }\end{array}$ & $\begin{array}{c}\text { Yes: } \\
\text { Multi-database } \\
\text { search (no start } \\
\text { date to July } \\
\text { 2017). Reference } \\
\text { lists of included } \\
\text { studies were } \\
\text { checked. } \\
\text { Screening was } \\
\text { conducted by two } \\
\text { authors, with a } \\
\text { third author }\end{array}$ \\
\hline
\end{tabular}




\begin{tabular}{|c|c|c|c|c|}
\hline & & $\begin{array}{c}\text { evaluation of } \\
\text { reviewer } \\
\text { agreement. If } \\
\text { inclusion was } \\
\text { unclear a second } \\
\text { author was } \\
\text { consulted } \\
\end{array}$ & \begin{tabular}{|c} 
authors \\
independently, \\
with a third \\
author utilised for \\
consensus
\end{tabular} & $\begin{array}{l}\text { utilised for } \\
\text { consensus }\end{array}$ \\
\hline \begin{tabular}{|l} 
5. Were the \\
criteria for \\
appraising studies \\
appropriate?
\end{tabular} & $\begin{array}{c}\text { Yes: } \\
\text { PEDro scale was } \\
\text { used }\end{array}$ & $\begin{array}{c}\text { Yes: } \\
\text { PEDro scale was } \\
\text { used }\end{array}$ & $\begin{array}{c}\text { Yes: } \\
\text { PEDro scale \& } \\
\text { Cochrane } \\
\text { Collaboration } \\
\text { Risk-of- bias tool } \\
\text { were used }\end{array}$ & $\begin{array}{c}\text { Yes: } \\
\text { PEDro scale was } \\
\text { used }\end{array}$ \\
\hline $\begin{array}{l}\text { 6. Was critical } \\
\text { appraisal } \\
\text { conducted by two } \\
\text { or more } \\
\text { reviewers } \\
\text { independently? }\end{array}$ & $\begin{array}{c}\text { Yes: } \\
2 \text { authors } \\
\text { independently. } \\
\text { Disagreements } \\
\text { resolved by } \\
\text { consensus }\end{array}$ & $\begin{array}{l}\text { Yes: } \\
\text { Validated against } \\
\text { the score in the } \\
\text { PEDro database. } \\
\text { Discrepancy } \\
\text { resolved by } \\
\text { another reviewer }\end{array}$ & $\begin{array}{c}\text { Yes: } \\
2 \text { reviewers } \\
\text { independently. } \\
\text { Disagreements } \\
\text { resolved by } \\
\text { consensus }\end{array}$ & $\begin{array}{c}\text { Yes: } \\
2 \text { reviewers } \\
\text { (unclear if } \\
\text { independently). } \\
\text { Validated against } \\
\text { the score in the } \\
\text { PEDro database }\end{array}$ \\
\hline $\begin{array}{l}\text { 7. Were there } \\
\text { methods to } \\
\text { minimize errors in } \\
\text { data extraction? }\end{array}$ & $\begin{array}{l}\text { Yes: } \\
\text { Undertaken by } \\
\text { two reviewers } \\
\text { independently }\end{array}$ & $\begin{array}{l}\text { No: } \\
\text { Not reported }\end{array}$ & $\begin{array}{l}\text { Yes: } \\
\text { Undertaken by } \\
\text { two reviewers } \\
\text { independently }\end{array}$ & $\begin{array}{l}\text { Yes: } \\
\text { Undertaken by } \\
\text { two reviewers } \\
\text { independently }\end{array}$ \\
\hline $\begin{array}{l}\text { 8. Were the } \\
\text { methods used to } \\
\text { combine studies } \\
\text { appropriate? }\end{array}$ & $\begin{array}{c}\text { Yes: } \\
\text { Meta-analysis } \\
\text { using } \\
\text { standardised } \\
\text { effect sizes for } \\
\text { pre- and post-test } \\
\text { outcomes was } \\
\text { undertaken. The } \\
\text { exact model } \\
\text { method was } \\
\text { unclear. } \\
\text { Heterogeneity } \\
\text { was evaluated } \\
\text { using } \text { I }^{2}\end{array}$ & $\begin{array}{c}\text { Yes: } \\
\text { A random effects } \\
\text { meta-analysis } \\
\text { using SMD was } \\
\text { undertaken } \\
\text { comparing } \\
\text { intervention and } \\
\text { control group } \\
\text { outcomes. } \\
\text { Heterogeneity } \\
\text { was evaluated } \\
\text { using I }{ }^{2} \text { but a } \\
\text { sensitivity } \\
\text { analysis was not } \\
\text { reported }\end{array}$ & $\begin{array}{c}\text { Yes: } \\
\text { A random effects } \\
\text { meta-analysis } \\
\text { using SMD was } \\
\text { undertaken. Pre } \\
\text { and post-test } \\
\text { outcomes were } \\
\text { used to compare } \\
\text { between group } \\
\text { data. } \\
\text { "Double } \\
\text { counting" bias } \\
\text { was corrected. } \\
\text { Heterogeneity } \\
\text { was evaluated } \\
\text { using I² and } \\
\text { sensitivity with a } \\
\text { leave-one-out } \\
\text { analysis }\end{array}$ & \begin{tabular}{|} 
No: \\
Data synthesis of \\
between group \\
and /or within \\
group changes \\
was descriptive. A \\
meta-analysis was \\
discounted due to \\
assumed \\
heterogeneity \\
between studies \\
rather than \\
statistical findings \\
\end{tabular} \\
\hline $\begin{array}{l}\text { 9. Was the } \\
\text { likelihood of } \\
\text { publication bias } \\
\text { assessed? }\end{array}$ & $\begin{array}{l}\text { Yes: } \\
\text { Using the Eggers } \\
\text { regression test. }\end{array}$ & $\begin{array}{l}\text { N/A: } \\
\text { Insufficient } \\
\text { number of } \\
\text { publications to }\end{array}$ & $\begin{array}{l}\text { Yes: } \\
\text { Using a funnel } \\
\text { plot and Eggers } \\
\text { regression test }\end{array}$ & $\begin{array}{l}\text { No: } \\
\text { Not reported }\end{array}$ \\
\hline
\end{tabular}




\begin{tabular}{|c|c|c|c|c|}
\hline & & $\begin{array}{l}\text { carry out } \\
\text { assessment of } \\
\text { publication bias }\end{array}$ & & \\
\hline $\begin{array}{l}\text { 10. Were } \\
\text { recommendations } \\
\text { for policy and/or } \\
\text { practice } \\
\text { supported by the } \\
\text { reported data? }\end{array}$ & $\begin{array}{l}\text { Yes: } \\
\text { Use of } \\
\text { strengthening } \\
\text { exercise to } \\
\text { improve strength } \\
\text { for PwMS was } \\
\text { substantiated. } \\
\text { Evidence to } \\
\text { support strength } \\
\text { training to } \\
\text { improve fatigue, } \\
\text { quality of life and } \\
\text { function but not } \\
\text { gait, balance or } \\
\text { mood. } \\
\text { Limited practice } \\
\text { specific } \\
\text { recommendations }\end{array}$ & $\begin{array}{l}\text { Yes: } \\
\text { Use of } \\
\text { strengthening } \\
\text { exercise to improve } \\
\text { strength for PwMS, } \\
\text { consistent with } \\
\text { current guidelines, } \\
\text { was substantiated. } \\
\text { Limited practice } \\
\text { specific } \\
\text { recommendations }\end{array}$ & $\begin{array}{l}\text { Yes: } \\
\text { Use of } \\
\text { strengthening } \\
\text { exercise to improve } \\
\text { strength for PwMS } \\
\text { was substantiated. } \\
\text { Preliminary } \\
\text { evidence provided } \\
\text { to support strength } \\
\text { training to improve } \\
\text { gait. } \\
\text { Limited practice } \\
\text { specific } \\
\text { recommendations } \\
\end{array}$ & $\begin{array}{l}\text { Yes: } \\
\text { Use of } \\
\text { strengthening } \\
\text { exercise to } \\
\text { improve strength } \\
\text { for PwMS, } \\
\text { consistent with } \\
\text { current } \\
\text { guidelines, was } \\
\text { substantiated. } \\
\text { Data was } \\
\text { inconclusive } \\
\text { regarding the } \\
\text { impact of } \\
\text { strength training } \\
\text { on gait. } \\
\text { Limited practice } \\
\text { specific } \\
\text { recommendations }\end{array}$ \\
\hline $\begin{array}{l}\text { 11. Were the } \\
\text { specific directives } \\
\text { for new research } \\
\text { appropriate? }\end{array}$ & $\begin{array}{l}\text { Yes: } \\
\text { Recommendation } \\
\text { for future } \\
\text { research on the } \\
\text { use of strength } \\
\text { training with } \\
\text { more severe } \\
\text { PwMS }\end{array}$ & $\begin{array}{l}\text { Yes: } \\
\text { To investigate } \\
\text { progressive } \\
\text { resistance } \\
\text { training on the } \\
\text { upper body or } \\
\text { power training } \\
\text { elements for } \\
\text { PwMS }\end{array}$ & $\begin{array}{l}\text { Yes: } \\
\text { To investigate } \\
\text { strengthening for } \\
\text { the ankle, trunk } \\
\text { and upper limb } \\
\text { muscles, if } \\
\text { strength gains } \\
\text { correlate with } \\
\text { increases in } \\
\text { function and } \\
\text { quality of life or } \\
\text { the use of } \\
\text { strength training } \\
\text { with more severe } \\
\text { PwMS }\end{array}$ & $\begin{array}{l}\text { Yes: } \\
\text { To investigate } \\
\text { higher intensity } \\
\text { training /dose } \\
\text { response, } \\
\text { relationship of } \\
\text { strength to gait } \\
\text { outcomes or } \\
\text { types of strength } \\
\text { training } \\
\text { interventions } \\
\text { most relevant to } \\
\text { improving gait for } \\
\text { PwMS }\end{array}$ \\
\hline
\end{tabular}

Table note: PEDro = Physiotherapy Evidence Database; SMD = Standard Mean Difference; MS = multiple sclerosis; PwMS = people with multiple sclerosis; RCTs = randomised control trials; PICO = Population, Intervention, Control, Outcomes 


\section{$\underline{\text { Results }}$}

All systematic reviews included participants with a diagnosis of MS, presenting with mild to moderate MS equivalent to an Expanded Disability Status Scale (EDSS) of 6.5 or less (ie participants were all ambulatory).

\section{Assessment of quality}

Some 18 unique trials in total were included across the four systematic reviews (duplicate data sets used in several studies were either counted as one study or removed from the synthesis). All four review papers used the PEDro scale to assess for quality and risk of bias in the original studies. Manca et al (2019) also reported on the Cochrane Collaboration Risk-of-Bias tool (Higgins et al, 2021). The reviewers' interpretation of the PEDro scale was consistent between reviewers, except for Jørgensen et al (2017), who regularly scored differently to two or three other review papers. The PEDro scores (including discrepancies) are shown in Table 3. Taking these discrepancies into consideration, seven studies scored 6 or above (high quality), six studies scored between 5 and 6 (high/moderate quality) and five scored 4 to 5 (moderate quality). The criteria, which were not achieved by the included studies within the four systematic reviews, included the lack of blinding of therapists, participants and assessors.

Publication bias was assessed using the Eggers regression test by two reviews (Cruickshank et al, 2015; Manca et al, 2019). Only Manca et al (2019) reported a significant result supported by a funnel plot, which showed a lack of trials that were statistically non-significant.

Table 3. Individual studies utilised across the four review papers showing number of reviews the study was included in, PEDro scores and reported strength outcome changes

\begin{tabular}{|l|c|c|c|}
\hline \multicolumn{1}{|c|}{ Study } & $\begin{array}{c}\text { Included in number of } \\
\text { systematic reviews } \\
\text { (out of four reviewed) }\end{array}$ & $\begin{array}{c}\text { PEDro Scale } \\
\text { (including any } \\
\text { discrepancy) }\end{array}$ & $\begin{array}{c}\text { Change in } \\
\text { Strength } \\
\text { outcome }\end{array}$ \\
\hline Broekmans et al (2011) & 4 & 6 (5: Jørgensen) & ++ \\
\hline $\begin{array}{l}\text { Dalgas et al (2009; 2010a; } \\
\text { 2010b; 2013) }\end{array}$ & 4 & 6 (5: Jørgensen) & ++ \\
\hline Dodd et al (2011) & 4 & 8 (7: Jørgensen) & ++ \\
\hline $\begin{array}{l}\text { DeBolt and McCubbin } \\
\text { 2004) }\end{array}$ & 3 & 6 (5: Jørgensen ) & + \\
\hline Fimland et al (2010) & 3 & 4 (6: Jørgensen) & ++ \\
\hline $\begin{array}{l}\text { Kjølhede et al (2015a; } \\
\text { 2015b) }\end{array}$ & 2 & 6 (5: Jørgensen ) & ++ \\
\hline Medina Perez et al (2014) & 2 & 6 & ++ \\
\hline Moradi et al (2015) & 2 & 5 & ++ \\
\hline Romberg et al (2004) & 2 & $6 / 6$ & + \\
\hline Sangelaji et al (2016) & & 5 & + \\
\hline
\end{tabular}




\begin{tabular}{|l|c|c|c|}
\hline Eftekhari et al (2012) & 1 & 5 & ++ \\
\hline Frevel and Maurer (2015) & 1 & 6 & $\mathrm{NC}$ \\
\hline Harvey et al (1999) & 1 & 6 & $\mathrm{NC}$ \\
\hline Hayes et al (2011) & 1 & 5 & + \\
\hline Learmonth et al (2012) & 1 & 7 & $\mathrm{NC}$ \\
\hline Manca et al (2017) & 1 & 7 & + \\
\hline $\begin{array}{l}\text { Medina -Perez et al } \\
\text { (2016) }\end{array}$ & 1 & 5 & $\mathrm{NC}$ \\
\hline Sabapathy et al (2011) & 1 & 5 & + \\
\hline
\end{tabular}

Table notes: + = significant improvement in one strength outcome from baseline to follow; ++ = statistical significant improvement in one strength outcome comparing intervention to control group (difference within difference); $N C=$ no significant improvement in any comparison; PEDro = Physiotherapy Evidence Database

\section{Strength outcomes}

All four reviews reported statistically and clinically significant improvements in strength outcomes following strength training, as shown in Table 4, either through a meta-analysis or descriptive analysis. Out of the 18 individual studies, only four showed no improvement in strength outcomes (as shown in Table 4).

Table 4. Summary Findings from Review Papers on Strength Outcomes Following Strength Training

\begin{tabular}{|c|c|c|}
\hline \multirow[t]{2}{*}{ Review paper } & \multicolumn{2}{|c|}{ Synthesis Method } \\
\hline & $\begin{array}{l}\text { Meta-analysis } \\
\text { SMD (95\%Cl) }\end{array}$ & $\begin{array}{l}\text { Descriptive analysis } \\
\text { (Vote Counting) }\end{array}$ \\
\hline $\begin{array}{c}\text { Cruickshank et al } \\
\text { (2015) }\end{array}$ & $\begin{array}{c}\text { RCTs=5, Non } \mathrm{RCTs}=1 \\
\text { Data sets }=10 \\
\text { SMD }=0.31(0.15-0.48) \\
\text { Statistically significant } \\
\text { Small effect* } \\
I^{2}=0 \%\end{array}$ & \\
\hline $\begin{array}{l}\text { Jørgensen et al } \\
\text { (2017) }\end{array}$ & $\begin{array}{c}\text { RCTs }=6 \\
\text { Data sets }=6 \\
\text { SMD }=0.45(0.18-0.72) \\
\text { Statistically significant } \\
\text { Moderate effect* } \\
I^{2}=0 \%\end{array}$ & \\
\hline $\begin{array}{l}\text { Mañago et al } \\
\text { (2019) }\end{array}$ & & $\begin{array}{l}\text { 10/13 studies showed } \\
\text { significant between groups } \\
\text { and/or within group } \\
\text { improvements }\end{array}$ \\
\hline
\end{tabular}




\begin{tabular}{|c|c|c|}
\hline & & $\begin{array}{c}\text { 6/13 studies showing } \\
\text { significant improvement } \\
\text { between groups. }\end{array}$ \\
\hline $\begin{array}{c}\text { Manca et al } \\
\text { (2019) }\end{array}$ & $\begin{array}{c}\text { RCTs=9 } \\
\text { Data sets }=14 \\
\text { SMD=0.37 (0.16-0.57) } \\
\text { Statistically significant } \\
\text { Small effect* } \\
I^{2}=21 \%\end{array}$ & \\
\hline
\end{tabular}

Table notes: *Effect size referenced against Schünemann et al (2021)

\section{How findings support current guidelines}

Reporting of individual study intervention characteristics was variable across the 18 studies and four reviews. Therefore, information about each guideline aspect was not available from every individual study. This commentary information is based on the information extracted from the four review papers and, as such, information has not been cross-checked back to the original studies.

There was no clear association between varying modifiable exercise factors and the increase in number of studies reporting a positive increase in strength outcomes. The majority of studies that used similar exercise recommendation variables to those discussed in the guidelines found a positive increase in strength outcomes. A descriptive evaluation, based on available information, of the strength outcomes from the individual studies in relation to the guideline recommendations is shown in Table 5.

Table 5. Evaluation of the strength outcomes from the individual studies in relation to the guideline recommendations

\begin{tabular}{|c|c|c|c|}
\hline & Guideline Overview & Study breakdown & Strength Outcomes \\
\hline \multirow[t]{2}{*}{$\begin{array}{l}\text { Muscle group } \\
\text { focus }\end{array}$} & \multirow[t]{2}{*}{ Major muscle groups } & $\begin{array}{l}18 / 18 \text { studies included } \\
\text { lower limb muscle } \\
\text { groups with } 17 / 18 \\
\text { reporting on lower limb } \\
\text { outcomes }\end{array}$ & $\begin{array}{l}\text { Lower limb: } \\
14 / 17=\text { positive }\end{array}$ \\
\hline & & $\begin{array}{l}8 / 18 \text { focused on whole } \\
\text { body or did not specify } \\
\text { with } 1 / 18 \text { reporting an } \\
\text { upper limb outcome }\end{array}$ & $\begin{array}{l}\text { Upper limb: } \\
1 / 1=\text { no change }\end{array}$ \\
\hline \multirow[t]{2}{*}{$\begin{array}{l}\text { Number of } \\
\text { exercises }\end{array}$} & \multirow[t]{2}{*}{$5-10$} & $\begin{array}{l}9 / 18 \text { studies included } 1- \\
4 \text { exercises }\end{array}$ & $\begin{array}{l}\text { 1-4 exercises: } \\
7 / 9=\text { positive }\end{array}$ \\
\hline & & $\begin{array}{l}7 / 18 \text { studies included } 5- \\
8 \text { exercises }\end{array}$ & $\begin{array}{l}\text { 5-8 exercises: } \\
6 / 7=\text { positive }\end{array}$ \\
\hline Frequency & $2-3 \times$ week & $\begin{array}{l}\text { 14/18 studies used a } \\
\text { frequency between 2-3 } \\
\text { x week }\end{array}$ & $\begin{array}{l}\text { 2-3 } \times \text { week: } \\
\text { 12/14= positive }\end{array}$ \\
\hline
\end{tabular}




\begin{tabular}{|c|c|c|c|}
\hline & & $\begin{array}{l}2 / 18 \text { studies used a } \\
\text { frequency between 3-5 } \\
x \text { week }\end{array}$ & $\begin{array}{l}3-5 x \text { week: } \\
2 / 2=\text { positive }\end{array}$ \\
\hline \multirow[t]{2}{*}{ Repetitions } & \multirow[t]{2}{*}{ 8-15 repetitions (reps) } & $\begin{array}{l}14 / 18 \text { studies used a } \\
\text { range which in part fell } \\
\text { into the guideline range } \\
\text { reporting between } 5-15 \\
\text { reps. }\end{array}$ & $\begin{array}{l}\text { 5-15 reps: } \\
\text { 11/14 = positive }\end{array}$ \\
\hline & & $\begin{array}{l}2 / 18 \text { studies used a } \\
\text { specific } 4 \text { reps }\end{array}$ & $\begin{array}{l}4 \text { reps: } \\
2 / 2=\text { positive }\end{array}$ \\
\hline \multirow[t]{2}{*}{ Sets } & \multirow[t]{2}{*}{$1-3$} & $\begin{array}{l}12 / 18 \text { studies used } \\
\text { between } 1-3 \text { sets }\end{array}$ & $\begin{array}{l}\text { 1-3 sets: } \\
\text { 10/12 = positive }\end{array}$ \\
\hline & & $\begin{array}{l}4 / 18 \text { studies used } \\
\text { between } 3-5 \text { sets }\end{array}$ & $\begin{array}{l}3-5 \text { sets: } \\
3 / 4=\text { positive }\end{array}$ \\
\hline \multirow[t]{4}{*}{ Resistance } & \multirow[t]{4}{*}{$\begin{array}{l}\text { Barely but safely finish } \\
\text { 8-15 reps }\end{array}$} & $\begin{array}{l}\text { 4/18 studies included a } \\
\text { range between } 50- \\
80 \% 1 \mathrm{RM} \text { with a further } \\
\text { 2/18 included 35- } \\
70 \% 1 \mathrm{RM} \text {. }\end{array}$ & $\begin{array}{l}\text { 35-80\%1RM: } \\
6 / 6=\text { positive }\end{array}$ \\
\hline & & $1 / 18$ using $85-90 \% 1 \mathrm{RM}$ & $\begin{array}{l}\text { 85-90\%1RM } \\
\text { 1/1= positive }\end{array}$ \\
\hline & & $\begin{array}{l}3 / 18 \text { studies included } 6- \\
15 \mathrm{RM} \text { with } 3 / 3 \\
\text { reporting a positive } \\
\text { strength outcome. }\end{array}$ & $\begin{array}{l}\text { 6-15RM: } \\
\text { 3/3=positive }\end{array}$ \\
\hline & & \multicolumn{2}{|c|}{$\begin{array}{l}3 / 4 \text { studies which did not identify a change in } \\
\text { strength outcome did not report a resistance } \\
\text { measure and } 1 / 4 \text { used the Borg scale. }\end{array}$} \\
\hline \multirow[t]{3}{*}{$\begin{array}{l}\text { Suggested } \\
\text { Modalities }\end{array}$} & \multirow{3}{*}{$\begin{array}{l}\text { Weight machines, free } \\
\text { weights, resistance } \\
\text { bands }\end{array}$} & $\begin{array}{l}\text { 10/18 studies used } \\
\text { weight machines }\end{array}$ & $\begin{array}{l}\text { Weight machines: } \\
10 / 10=\text { positive }\end{array}$ \\
\hline & & $\begin{array}{l}1 / 18 \text { studies used body } \\
\text { weight exercises alone } \\
\text { and } 1 / 18 \text { used free } \\
\text { weights alone }\end{array}$ & $\begin{array}{l}\text { Body weight alone: } \\
1 / 1=\text { no change } \\
\text { Free weights alone: } \\
1 / 1=\text { no change }\end{array}$ \\
\hline & & $\begin{array}{l}\text { 5/18 studies used } \\
\text { combined training using } \\
\text { body weight exercises } \\
\text { and another modality } \\
\text { (Resistance bands, } \\
\text { weighted vest, weight } \\
\text { machine) }\end{array}$ & $\begin{array}{l}\text { Combined training: } \\
3 / 5=\text { positive }\end{array}$ \\
\hline Rest & $\begin{array}{l}\text { 1-4 mins between sets } \\
\text { and exercises. }\end{array}$ & $\begin{array}{l}\text { Only } 5 / 18 \text { studied } \\
\text { reported on rest with } \\
\text { variation between } 0.5-3 \\
\text { mins }\end{array}$ & $\begin{array}{l}\text { Rest period: } \\
4 / 5=\text { positive }\end{array}$ \\
\hline
\end{tabular}




\begin{tabular}{|c|c|c|c|}
\hline \multirow[t]{3}{*}{ Location } & \multirow[t]{3}{*}{$\begin{array}{l}\text { Not reported in the } \\
\text { guidelines }\end{array}$} & $\begin{array}{l}3 / 18 \text { studies were home } \\
\text { based }\end{array}$ & $\begin{array}{l}\text { Home based: } \\
2 / 3=\text { positive }\end{array}$ \\
\hline & & $\begin{array}{l}3 / 18 \text { identified they } \\
\text { were based in a gym / } \\
\text { rehab centre with a } \\
\text { further } 9 / 18 \text { not } \\
\text { providing a location but } \\
\text { using weight machines } \\
\text { suggesting a centre } \\
\text { based exercise }\end{array}$ & $\begin{array}{l}\text { Assumed gym based: } \\
11 / 12 \text { = positive }\end{array}$ \\
\hline & & $\begin{array}{l}1 / 18 \text { was a community } \\
\text { class without equipment }\end{array}$ & $\begin{array}{l}\text { Community class (no } \\
\text { equipment): } \\
1 / 1=\text { no change }\end{array}$ \\
\hline \multirow[t]{2}{*}{ Supervision } & \multirow[t]{2}{*}{$\begin{array}{c}\text { Supervised exercise is } \\
\text { advisable but } \\
\text { not essential }\end{array}$} & $\begin{array}{l}7 / 18 \text { were reported as } \\
\text { supervised with a } \\
\text { further } 6 \text { likely } \\
\text { supervised as centre } \\
\text { based or using } \\
\text { machines. }\end{array}$ & $\begin{array}{l}\text { Assumed supervision: } \\
11 / 13=\text { positive }\end{array}$ \\
\hline & & $\begin{array}{l}1 / 18 \text { independent with a } \\
\text { further } 2 \text { likely } \\
\text { independent as home } \\
\text { based. }\end{array}$ & $\begin{array}{l}\text { Assumed unsupervised: } \\
2 / 3=\text { positive }\end{array}$ \\
\hline Progression & $\begin{array}{l}\text { Gradually progress } \\
\text { duration, frequency and } \\
\text { intensity (suggests } \\
\text { progress intensity last) }\end{array}$ & \multicolumn{2}{|c|}{ Not evaluated from the study characteristics } \\
\hline $\begin{array}{l}\text { Joint aerobic } \\
\text { and resistance } \\
\text { training }\end{array}$ & $\begin{array}{l}\text { Can be performed on } \\
\text { the same day }\end{array}$ & \multicolumn{2}{|c|}{ Not evaluated from the study characteristics } \\
\hline
\end{tabular}

Note: Positive change = significant improvement in one or more strength outcomes (baseline to follow-up or between groups)

\section{Secondary outcomes}

There is some evidence that strength training, compared to no intervention/waiting list, will improve some gait outcomes, with Manca et al (2019) reporting that the pooled results from the metaanalysis show statistically significant improvements in walking speed. Mañago et al (2019) reported that six out of 13 studies demonstrated a significant improvement in gait; this is in comparison with Cruickshank et al (2015), who reported that none of the four studies evaluated reported significant improvements in functional mobility. Limited findings can be drawn regarding other outcomes, due to the small number of studies involved, although there is a suggestion that fatigue can be improved with strength training (Cruickshank et al, 2015). 
Table 6. Summary Findings from Review Papers on Secondary Outcomes Following Strength Training

\begin{tabular}{|c|c|c|c|}
\hline \multirow[t]{2}{*}{ Review Paper } & \multirow[t]{2}{*}{ Outcome } & \multicolumn{2}{|c|}{ Synthesis Method } \\
\hline & & $\begin{array}{l}\text { Meta-analysis } \\
\text { (SMD (95\%Cl) }\end{array}$ & $\begin{array}{l}\text { Descriptive Analysis } \\
\text { (Vote Counting) }\end{array}$ \\
\hline \multirow[t]{7}{*}{$\begin{array}{l}\text { Cruickshank et al } \\
\text { (2015) }\end{array}$} & Functional mobility & & $\begin{array}{l}\text { 0/4 found significant } \\
\text { improvement }\end{array}$ \\
\hline & Balance & & $\begin{array}{l}1 / 3 \text { found significant } \\
\text { improvement }\end{array}$ \\
\hline & Functional Capacity & & $\begin{array}{l}1 / 1 \text { found significant } \\
\text { improvement }\end{array}$ \\
\hline & Quality of Life (QoL) & & $\begin{array}{l}2 / 3 \text { found significant } \\
\text { improvement }\end{array}$ \\
\hline & Fatigue & & $\begin{array}{l}3 / 3 \text { found significant } \\
\text { improvement }\end{array}$ \\
\hline & Mood & & $\begin{array}{l}1 / 2 \text { found significant } \\
\text { improvement }\end{array}$ \\
\hline & Muscle endurance & & $\begin{array}{l}1 / 2 \text { found significant } \\
\text { improvement }\end{array}$ \\
\hline Mañago et al (2019) & Gait Outcomes & & $\begin{array}{l}6 / 13 \text { found significant } \\
\text { improvements }\end{array}$ \\
\hline \multirow[t]{4}{*}{ Manca et al (2019) } & Walking speed & $\begin{array}{c}\text { RCTs }=6 \\
\text { Data sets }=6 \\
\text { SMD }=0.35(0.07-0.63) \\
\text { Small effect } * \\
I^{2}=0 \%\end{array}$ & \\
\hline & 2 Minute Walk Test & $\begin{array}{c}\text { RCTs }=2 \\
\text { Data sets }=2 \\
\text { SMD }=0.5(-0.48-1.48) \\
\text { Moderate effect* } \\
I^{2}=23 \%\end{array}$ & \\
\hline & $\begin{array}{l}\text { Timed-Up-and-Go } \\
\text { (TUG) }\end{array}$ & $\begin{array}{c}\text { RCTs }=2 \\
\text { Data sets }=2 \\
\text { SMD }=0.38(-0.16-0.91) \\
\text { Non-significant } \\
1^{2}=0 \%\end{array}$ & \\
\hline & 6 Minute Walk Test & $\begin{array}{c}\text { RCTs }=2 \\
\text { Data sets }=2 \\
\text { SMD }=-0.25(-0.3-0.8) \\
\text { Non-significant } \\
I^{2}=0 \%\end{array}$ & \\
\hline
\end{tabular}


Note: *Effect size referenced against Schünemann et al (2021)

\section{Discussion}

Using the JBI critical appraisal checklist for systematic reviews and research syntheses (Aromataris et al, 2015), it was judged that the systematic reviews by Manca et al (2019) and Cruickshank et al (2015) had used appropriate methods for all 11 criteria. The review by Mañago et al (2019) met nine criteria, as it did not undertake appropriate methods of synthesis. The review classified the included studies to be heterogeneous and hence did not perform a meta-analysis. However, this heterogeneity was not evaluated statistically and, considering the other three reviews achieved a meta-analysis with at least some of the included studies, it could be argued that Mañago et al (2019) should have included a meta-analysis of some of the study information. The review by Jørgensen et al (2017) met eight criteria, due to the lack of detail around the search strategy and data extraction. Both Cruickshank et al (2015) and Manca et al (2019) made an error in including multiple outcomes measuring strength from a single study as different effect estimates from the same sample are typically correlated and assuming independence may be inappropriate (Sutton et al, 2000).

Subsequently, this means that the confidence intervals presented in these reviews are questionable, although they are similar to the review by Jørgensen et al (2017), where this error did not occur. It is worth noting that, while Manca et al (2019) and Mañago et al (2019) identified a comprehensive search strategy, they did limit the search to English language studies, and Cruickshank et al (2015) did not provide detail of the study screening process. However, in the context of this work, the four reviews were considered together, which decreases the risk of reduced recall. Therefore, they can be considered to provide an adequate and comprehensive summary of evidence relating to strength training for PwMS. Additionally, It is important to acknowledge that this is a commentary and the original focus was not to identify all reviews in this area, but instead to gather a substantial number of reviews addressing the topic area to inform practice.

At a study level, all studies were assessed by the review papers as being of moderate to high quality. The main methodological concerns were around the lack of blinding of therapists, participants and assessors, which, in part, reflects the intervention being investigated. None of the four systematic reviews carried out a meta-regression or subgroup analysis for quality of evidence but, based on the evidence being judged to be of moderate to high quality, the effect estimates within the reviews are unlikely to be substantially affected by bias.

Current evidence for strength training for PwMS is focused on those living with mild to moderate disease (ie they are ambulatory). For this group of PwMS, strength training is associated with improved muscle strength and, therefore, has a clear role in both clinical practice for people presenting with muscle weakness and in general fitness. What remains less clear is the role strength training plays in other functional outcomes, such as walking, functional activity and quality of life. Muscle weakness has been shown to be associated with reduction in a variety of functional tasks (Jørgensen et al, 2017) and is commonly identified as an underlying modifiable impairment in clinical practice. These more functional outcomes may also be more relevant to PwMS than a pure strength outcome. Some weak evidence suggests that strength training can be used to improve aspects of gait performance; however, the evidence for other outcomes is too limited currently to draw conclusions for practice.

Both guidelines broadly sit within the intervention parameters of the studies documented, and these were generally associated with positive results (Latimer-Cheung et al, 2013b; Kim et al, 2019). Therefore, these recommendations should be adopted as part of standard practice where relevant. See Table 7 for full considerations for practice in relation to current guidelines. 
Table 7. Considerations for practice in relation to current guidelines

\begin{tabular}{|c|c|}
\hline $\begin{array}{l}\text { Guideline overview (Latimer-Cheung et al, } \\
\text { (2013b; Kim et al, 2019) }\end{array}$ & Considerations for practice \\
\hline Muscle group focus = major muscle groups & $\begin{array}{l}\text { Current evidence supports strength training for } \\
\text { lower limbs rather than all major muscle } \\
\text { groups, although this is due to a lack of studies } \\
\text { addressing upper limb training and evaluating } \\
\text { upper limb outcome measures }\end{array}$ \\
\hline Number of exercises $=5-10$ & $\begin{array}{l}\text { Current evidence supports between 1-8 } \\
\text { exercises, but reflects that some of the studies } \\
\text { were working specific muscle groups only }\end{array}$ \\
\hline Frequency $=2-3 \times$ week & $\begin{array}{l}\text { Current evidence supports that the frequency } \\
\text { of exercise should be between } 2-3 \text { times a week }\end{array}$ \\
\hline Repetitions $=8-15$ & $\begin{array}{l}\text { Current evidence supports guideline with } \\
\text { suggestion that a larger reps range may be } \\
\text { acceptable, eg 5-15 }\end{array}$ \\
\hline Sets $=1-3$ & $\begin{array}{l}\text { Current evidence supports that } 1-3 \text { sets are } \\
\text { adequate to produce strength improvements }\end{array}$ \\
\hline $\begin{array}{l}\text { Resistance }=\text { barely but safely finish } 8-15 \\
\text { reps }(8-15 R M)\end{array}$ & $\begin{array}{l}\text { Current evidence difficult to interpret due to } \\
\text { the variety of outcome measures used in the } \\
\text { studies. Use of percentage of } 1 \mathrm{RM} \text { is difficult to } \\
\text { translate to clinical practice }\end{array}$ \\
\hline $\begin{array}{l}\text { Suggested modalities }=\text { weight machines, free } \\
\text { weights, resistance bands }\end{array}$ & $\begin{array}{l}\text { The majority of evidence is for weight machines } \\
\text { rather than other modalities, which may not be } \\
\text { accessible in practice. It is unclear how } \\
\text { transferable the evidence from weight } \\
\text { machines is to other forms of resistance }\end{array}$ \\
\hline Rest $=1-4$ mins between sets and exercises & Poorly reported in current evidence \\
\hline Location $=$ not reported in the guidelines & $\begin{array}{l}\text { Poorly reported in current evidence. Although } \\
\text { assuming machine-based exercise is centre- } \\
\text { based, the majority of evidence is for centre- } \\
\text { based strength training, which may not be } \\
\text { accessible in practice }\end{array}$ \\
\hline $\begin{array}{l}\text { Supervision= supervised exercise is advisable } \\
\text { but not essential }\end{array}$ & $\begin{array}{l}\text { Poorly reported in current evidence. Although } \\
\text { assuming centre-based exercise as supervised } \\
\text { and home exercise as independent, there is a } \\
\text { larger evidence base for supervised exercises } \\
\text { supporting the guidelines }\end{array}$ \\
\hline $\begin{array}{l}\text { Progression = gradually progress duration, } \\
\text { frequency and intensity (suggests progress } \\
\text { intensity last) }\end{array}$ & Poorly reported in current evidence \\
\hline $\begin{array}{l}\text { Joint aerobic and resistance training= Can be } \\
\text { performed on the same day }\end{array}$ & Not reported in current evidence \\
\hline
\end{tabular}


Historically, PwMS were advised not to exercise (Sutherland and Andersen, 2001) and, while exercise is now an established part of MS treatment, PwMS have been predominately involved in aerobic training (Manca et al, 2019). Considering this, it is likely that both PwMS and the health and exercise professionals with whom they work with may have a knowledge gap around strength training. A recent report into strength training (Chartered Society of Physiotherapy, 2020) identified that people living with long-term conditions continue to hold the belief that their condition prevents them from engaging in strength training. This reinforces the need for education about strength training for people with long-term conditions and professionals who work with them.

Questions remain around elements of best practice for strengthening exercise for PwMS. Future research needs to further evaluate the impact of strength training with a focus on: (1) other key outcomes, such as fatigue, function and quality of life; (2) people with severe MS (an EDSS greater than 6.5); and (3) upper limb strength. More pragmatic studies are also required that investigate aspects of strength training in situations relevant to PwMS when access to weight machines, centrebased exercise, identification of 1RM and supervision may be limited. Additionally, clinicians, exercise professionals and PwMS would benefit from knowledge and understanding about both the use of rest periods and exercise progression to maximise training effect for PwMS. Finally, as the most recent paper in these four reviews is from 2017, there is a growing need for these reviews to be updated to take emerging evidence into consideration.

Declaration of interest: This report is independent research funded by the National Institute for Health Research Applied Research Collaboration North West Coast (ARC NWC). The views expressed in this publication are those of the author(s) and not necessarily those of the National Institute for Health Research, the NHS or the Department of Health and Social Care.

\section{KEY POINTS}

- Strength training is associated with improved muscle strength in people with mild to moderate (Expanded Disability Status Scale [EDSS] of 6.5 or less) multiple sclerosis

- The effectiveness of strength training for functional outcomes are inconclusive

- Further research is required in regard to the effectiveness of strength and conditioning on functional-related outcomes.

\section{CPD REFLECTIVE QUESTIONS}

- What are the main limitations of the for systematic reviews included in this commentary?

- What knowledge gaps do you have around strength and conditioning for people with multiple sclerosis (PwMS)?

- What current strength and conditioning training do you use with PWMS, and do you use any of the principles identified in the guidelines?

\section{References}

Amatya B, Khan F, Galea M. Rehabilitation for people with multiple sclerosis: an overview of Cochrane Reviews. Cochrane Database Syst Rev. 2019;1(1):CD012732.

https://doi.org/10.1002/14651858.CD012732.pub2 
Aromataris E, Fernandez R, Godfrey C, Holly C, Kahlil H, Tungpunkom P. Summarizing systematic reviews: methodological development, conduct and reporting of an umbrella review approach. International Journal of Evidence Based Healthcare, 2015;13(3), 132-140.

Broekmans T, Roelants M, Feys P, Alders G, Gijbels D, Hanssen I, Stinissen P, Eijnde, B. Effects of long-term resistance training and simultaneous electro-stimulation on muscle strength and functional mobility in multiple sclerosis. Multiple sclerosis (Houndmills, Basingstoke, England), 2011;17(4), 468-477. https://doi.org/10.1177/1352458510391339

Charted Society of Physiotherapy. Strength messaging Insight: Full findings from the research. 2020; Retrieved from

https://www.csp.org.uk/system/files/publication files/CSP Strength\%20Messaging\%20Insight Full \%20Research\%20Findings December\%202020\%20\%28for\%20publication\%29.pdf

Cruickshank T, Reyes A, Ziman (2015). A systematic review and meta-analysis of strength training in individuals with multiple sclerosis or Parkinson disease. Medicine, 2015;94(4), 1-15. https://doi.org/10.1097/MD.0000000000000411

Dalgas U, Stenager E, Jakobsen J, Petersen T, Hansen H, Knudsen C, Overgaard K, Ingemann-Hansen $\mathrm{T}$. Resistance training improves muscle strength and functional capacity in multiple sclerosis. Neurology, 2009;73(18), 1478-1484. https://doi.org/10.1212/WNL.0b013e3181bf98b4

Dalgas U, Stenager E, Jakobsen J, Petersen T, Hansen H, Knudsen C, Overgaard K, Ingemann-Hansen, $T$. Fatigue, mood and quality of life improve in MS patients after progressive resistance training. Multiple sclerosis (Houndmills, Basingstoke, England), 2010;16(4), 480-490. https://doi.org/10.1177/1352458509360040

Dalgas U, Stenager E, Jakobsen J, Petersen T, Overgaard K, Ingemann-Hansen T. Muscle fiber size increases following resistance training in multiple sclerosis. Multiple sclerosis (Houndmills, Basingstoke, England), 2010;16(11), 1367-1376. https://doi.org/10.1177/1352458510377222

Dalgas U, Stenager E, Lund, C, Rasmussen C, Petersen T, Sørensen H, Ingemann-Hansen T, Overgaard $\mathrm{K}$. Neural drive increases following resistance training in patients with multiple sclerosis. Journal of neurology, 2013;260(7), 1822-1832. https://doi.org/10.1007/s00415-013-6884-4

DeBolt L, McCubbin J. The effects of home-based resistance exercise on balance, power, and mobility in adults with multiple sclerosis. Archives of physical medicine and rehabilitation, 2004;85(2), 290-297. https://doi.org/10.1016/j.apmr.2003.06.003

Dodd K, Taylor N, Shields N, Prasad D, McDonald E, Gillon A. Progressive resistance training did not improve walking but can improve muscle performance, quality of life and fatigue in adults with multiple sclerosis: a randomized controlled trial. Multiple sclerosis (Houndmills, Basingstoke, England), 2011;17(11), 1362-1374. https://doi.org/10.1177/1352458511409084

Eftekhari E, Mostahfezian M, Etemadifar M,Zafari A. Resistance training and vibration improve muscle strength and functional capacity in female patients with multiple sclerosis. Asian journal of sports medicine, 2012;3(4), 279-284. https://doi.org/10.5812/asjsm.34552

Fimland M, Helgerud J, Gruber M, Leivseth G, Hoff J. Enhanced neural drive after maximal strength training in multiple sclerosis patients. European journal of applied physiology, 2010;110(2), 435-443. https://doi.org/10.1007/s00421-010-1519-2 
Frevel D, Mäurer M. Internet-based home training is capable to improve balance in multiple sclerosis: a randomized controlled trial. European journal of physical and rehabilitation medicine, 2015;51(1), 23-30.

Harvey L, Smith D, Jones R. The effect of weighted leg raises on quadriceps strength, EMG parameters and functional activities in people with multiple sclerosis. Physiotherapy, 1999;85(3), 154-161.

Hayes H, Gappmaier E, LaStayo P. Effects of high-intensity resistance training on strength, mobility, balance, and fatigue in individuals with multiple sclerosis: a randomized controlled trial. Journal of neurologic physical therapy : JNPT, 2011;35(1), 2-10.

https://doi.org/10.1097/NPT.0b013e31820b5a9d

Higgins JPT, Thomas J, Chandler J, Cumpston M, Li T, Page MJ, Welch VA (editors) . Cochrane Handbook for Systematic Reviews of Interventions version 6.2 (updated February 2021). Cochrane. 2021. Available from www.training.cochrane.org/handbook.

Jones C, Pohar S, Warren S, Turpin K, Warren K. The burden of multiple sclerosis: a community health survey. Health and quality of life outcomes, 2008;6, 1. https://doi.org/10.1186/1477-7525-61

Jørgensen M, Dalgas U, Wens I, Hvid L. Muscle strength and power in persons with multiple sclerosis - A systematic review and meta-analysis. Journal of the neurological sciences, 2017;376, 225-241. https://doi.org/10.1016/j.jns.2017.03.022

Kim Y, Lai B, Mehta T, Thirumalai M, Padalabalanarayanan S, Rimmer J.,Motl R. Exercise Training Guidelines for Multiple Sclerosis, Stroke, and Parkinson Disease: Rapid Review and Synthesis. American journal of physical medicine \& rehabilitation, 2019;98(7), 613-621. https://doi.org/10.1097/PHM.0000000000001174

Kinnett-Hopkins D, Adamson B, Rougeau K, Motl R. People with MS are less physically active than healthy controls but as active as those with other chronic diseases: An updated metaanalysis. Multiple sclerosis and related disorders, 2017;13, 38-43. https://doi.org/10.1016/j.msard.2017.01.016

Kjølhede T, Vissing K, Dalgas U. Multiple sclerosis and progressive resistance training: a systematic review. Multiple sclerosis (Houndmills, Basingstoke, England), 2012;18(9), 1215-1228. https://doi.org/10.1177/1352458512437418

Kjølhede T, Vissing K, de Place L, Pedersen B, Ringgaard S, Stenager E, Petersen T, Dalgas U. Neuromuscular adaptations to long-term progressive resistance training translates to improved functional capacity for people with multiple sclerosis and is maintained at follow-up. Multiple sclerosis (Houndmills, Basingstoke, England), 2015;21(5), 599-611.

https://doi.org/10.1177/1352458514549402

Kjølhede T, Dalgas U, Gade A, Bjerre M, Stenager E, Petersen T, Vissing K. Acute and chronic cytokine responses to resistance exercise and training in people with multiple sclerosis. Scandinavian journal of medicine \& science in sports, 2016;26(7), 824-834. https://doi.org/10.1111/sms.12504

Kobelt G, Thompson A, Berg J, Gannedahl M, Eriksson J. New insights into the burden and costs of multiple sclerosis in Europe. Multiple Sclerosis Journal, 2017;23(8), 1123-1136.

https://doi.org/10.1177/1352458517694432 
Latimer-Cheung A, Pilutti L, Hicks A, Martin Ginis K, Fenuta A, MacKibbon K, Motl R. Effects of exercise training on fitness, mobility, fatigue, and health-related quality of life among adults with multiple sclerosis: a systematic review to inform guideline development. Archives of physical medicine and rehabilitation, 2013a;94(9), 1800-1828.e3.

https://doi.org/10.1016/j.apmr.2013.04.020

Latimer-Cheung, A, Martin Ginis K, Hicks A, Motl R, Pilutti L, Duggan M, Wheeler G, Persad R, Smith, $K$. Development of evidence-informed physical activity guidelines for adults with multiple sclerosis. Archives of physical medicine and rehabilitation, 2013b;94(9), 1829-1836.e7. https://doi.org/10.1016/i.apmr.2013.05.015

Learmonth Y, Paul L, Miller L, Mattison P, McFadyen A. The effects of a 12-week leisure centrebased, group exercise intervention for people moderately affected with multiple sclerosis: a randomized controlled pilot study. Clinical rehabilitation, 2012;26(7), 579-593.

https://doi.org/10.1177/0269215511423946

Lunde H, Assmus J, Myhr K, Bø L, Grytten N. Survival and cause of death in multiple sclerosis: A 60year longitudinal population study Journal of Neurology, Neurosurgery \& Psychiatry, 2017;88,621-625. https://doi:10.1136/innp-2016$\underline{315238}$

Mañago M, Glick S, Hebert J, Coote S, Schenkman M. Strength Training to Improve Gait in People with Multiple Sclerosis: A Critical Review of Exercise Parameters and Intervention Approaches. International journal of MS care, 2019;21(2), 47-56. https://doi.org/10.7224/15372073.2017-079

Manca A, Cabboi M, Dragone D, Ginatempo F, Ortu E, De Natale E, Mercante B, Mureddu G, Bua G, Deriu F. Resistance Training for Muscle Weakness in Multiple Sclerosis: Direct Versus Contralateral Approach in Individuals With Ankle Dorsiflexors' Disparity in Strength. Archives of physical medicine and rehabilitation, 2017;98(7), 1348-1356.e1. https://doi.org/10.1016/j.apmr.2017.02.019

Manca A, Dvir Z, Deriu F. Meta-analytic and Scoping Study on Strength Training in People With Multiple Sclerosis. Journal of strength and conditioning research, 2019;33(3), 874-889. https://doi.org/10.1519/JSC.0000000000002381

Medina-Perez C, de Souza-Teixeira F, Fernandez-Gonzalo R, de Paz-Fernandez J. Effects of a resistance training program and subsequent detraining on muscle strength and muscle power in multiple sclerosis patients. NeuroRehabilitation, 2014;34(3), 523-530. https://doi.org/10.3233/NRE141062

Medina-Perez C, de Souza-Teixeira F, Fernandez-Gonzalo R, Hernandez-Murua J, Antonio de PazFernandez J. Effects of high-speed power training on muscle strength and power in patients with multiple sclerosis. Journal of rehabilitation research and development, 2016;53(3), 359-368. https://doi.org/10.1682/JRRD.2014.08.0186

Moradi M, Sahraian M, Aghsaie A, Kordi M, Meysamie A, Abolhasani M, Sobhani V. Effects of Eightweek Resistance Training Program in Men With Multiple Sclerosis. Asian journal of sports medicine, 2015;6(2), e22838. https://doi.org/10.5812/asjsm.6(2)2015.22838

Public Health England. [PHE]. Physical activity for general health benefits in disabled adults: Summary of a rapid evidence review for the UK Chief Medical Officers' update of the physical activity. Public health England. 2018. 
Public Health England. [PHE]. (2019). UK Chief Medical Officers' Physical Activity Guidelines

Public Health England. [PHE]. (2020). Multiple sclerosis: prevalence, incidence and smoking status data briefing. Retrieved from https://www.gov.uk/government/publications/multiple-sclerosisprevalence-incidence-and-smoking-status/multiple-sclerosis-prevalence-incidence-and-smokingstatus-data-briefing

Romberg A, Virtanen A, Ruutiainen J, Aunola S, Karppi S, Vaara M, Surakka J, Pohjolainen T, Seppänen $A$. Effects of a 6-month exercise program on patients with multiple sclerosis: a randomized study. Neurology, 2004;63(11), 2034-2038.

https://doi.org/10.1212/01.wnl.0000145761.38400.65

Sangelaji B, Kordi M, Banihashemi F, Nabavi S, Khodadadeh S, Dastoorpoor M. A combined exercise model for improving muscle strength, balance, walking distance, and motor agility in multiple sclerosis patients: A randomized clinical trial. Iranian journal of neurology, 2016;15(3), 111-120

Sabapathy N. , Minahan C, Turner G, Broadley S. Comparing endurance- and resistance-exercise training in people with multiple sclerosis: a randomized pilot study. Clinical rehabilitation, 2011;25(1), 14-24. https://doi.org/10.1177/0269215510375908

Schünemann H, Vist G, Higgins J, Santesso N, Deeks J, Glasziou P, Elie A, Akl E, Gordon H, Guyatt G. (2021). Interpreting results and drawing conclusions. In J.Higgins, J.Thomas, J.Chandler, M.Cumpston, T.Li, M.Page, \& V.Welch (Eds.), Cochrane handbook for systematic reviews of interventions. Retrieved from http://www.training.cochrane.org/handbook/current

Sutherland G, Andersen M. Exercise and multiple sclerosis: physiological, psychological, and quality of life issues. J Sports Med Phys Fitness. Dec2001;41(4):421-32. PMID: 11687760.

Sutton A, Abrams K, Jones D, Sheldon T, Song F. Methods for meta-analysis in medical research. Wiley \& Sons Ltd. Chichester; 2000

Walton, C, King R, Rechtman L, Kaye W, Leray E, Marrie R, Robertson N, La Rocca N, Uitdehaag, B, van der Mei I, Wallin M, Helme A, Angood Napier C, Rijke N, Baneke P. Rising prevalence of multiple sclerosis worldwide: Insights from the Atlas of MS, third edition. Multiple Sclerosis Journal, 2020;26(14), 1816-1821. https://doi.org/10.1177/1352458520970841 